\title{
Association between Admission Hyperglycemia and Culprit Lesion Characteristics in Nondiabetic Patients with Acute Myocardial Infarction: An Intravascular Optical Coherence Tomography Study
}

\author{
Jinying Zhou, ${ }^{1}$ Zhaoxue Sheng, ${ }^{1}$ Chen Liu, ${ }^{1}$ Peng Zhou, ${ }^{1}$ Jiannan Li, ${ }^{1}$ Runzhen Chen (D), \\ Li Song, ${ }^{1}$ Hanjun Zhao, ${ }^{1}$ and Hongbing Yan $\mathbb{1}^{1,2}$ \\ ${ }^{1}$ Department of Cardiology, Fuwai Hospital, National Center for Cardiovascular Diseases, Peking Union Medical College and Chinese \\ Academy of Medical Sciences, Beijing, China \\ ${ }^{2}$ Fuwai Hospital, Chinese Academy of Medical Sciences, Shenzhen, China
}

Correspondence should be addressed to Hongbing Yan; hbyanfuwai2018@163.com

Received 9 April 2020; Accepted 5 June 2020; Published 22 June 2020

Academic Editor: Yoshifumi Saisho

Copyright (c) 2020 Jinying Zhou et al. This is an open access article distributed under the Creative Commons Attribution License, which permits unrestricted use, distribution, and reproduction in any medium, provided the original work is properly cited.

\begin{abstract}
Background. Hyperglycemia is frequently observed in acute myocardial infarction (AMI). Diabetes mellitus (DM) patients and non-DM patients have different culprit lesion phenotypes and few data are available on non-DM patients with admission hyperglycemia. Therefore, we aimed to investigate the association between admission hyperglycemia and culprit lesion characteristics using optical coherence tomography (OCT) in AMI patients. Methods and Results. We consecutively enrolled 434 patients with AMI, and 277 patients were included in analysis: 65.7\% $(n=182)$ non-DM patients and $34.3 \%(n=95)$ DM patients. We measured acute blood glucose $(\mathrm{ABG})$ and hemoglobin $\mathrm{A}_{1 \mathrm{c}}$ to calculate the acute-to-chronic glycemic ratio (A/C). Then, we grouped non-DM patients into tertiles of A/C. OCT-based culprit lesion characteristics were compared across A/C tertiles in non-DM patients and between DM and non-DM patients. Non-DM patients had fewer lipid-rich plaques (52.7\% versus $68.4 \%, p=0.012)$ and thin-cap fibroatheroma (TCFA) $(19.8 \%$ versus $34.7 \%, p=0.006)$ than DM patients but similar prevalence of plaque rupture $(47.3 \%$ versus $56.8 \%, p=0.130)$. Non-DM patients with the highest $\mathrm{A} / \mathrm{C}$ tertile had the highest prevalence of plaque rupture $\left(p_{\text {for trend }}=0.002\right)$, lipid-rich plaque $\left(p_{\text {for trend }}=0.001\right)$, and TCFA $\left(p_{\text {for trend }}=0.003\right)$. A/C $>1.22$ but not $\mathrm{ABG}>140 \mathrm{mg} / \mathrm{dl}$ predicted a high prevalence of plaque rupture, lipid-rich plaque, and TCFA in non-DM patients. Conclusions. In AMI patients without DM, admission hyperglycemia is associated with vulnerable culprit lesion characteristics, and $\mathrm{A} / \mathrm{C}$ is a better predictor for vulnerable culprit plaque characteristics than $\mathrm{ABG}$. These results call for a tailored evaluation and management of glucose metabolism in nondiabetic AMI patients. This trial is registered with NCT03593928.
\end{abstract}

\section{Introduction}

Diabetes mellitus (DM) in general increases vascular complications, including coronary heart disease, ischemic stroke, and vascular deaths [1]. DM patients have a high prevalence of multivessel disease and an accelerated atherosclerotic progression related to the glucose level [2]. Optical coherence tomography (OCT) allows accurate evaluation of coronary atherosclerotic plaques in vivo. Although a pilot study including 63 participants with coronary artery diseases
(CAD) found no significant difference in plaque characteristics between the DM group and the non-DM group [3], recent studies reported conflicting results that diabetic individuals with $C A D$ may have more calcification $[4,5]$ or lipid-rich plaques [6-8]. Additionally, individuals with hemoglobin $\mathrm{A}_{1 \mathrm{c}}\left(\mathrm{HbA}_{1 \mathrm{c}}\right) \geq 8 \%$ had the highest prevalence of thin-cap fibroatheroma (TCFA) [6]. These results indicate that the glucose level has an impact on coronary plaque characteristics, but it remains unclear whether such impact exists in non-DM individuals with CAD. 
Acute hyperglycemia at admission is frequently observed in acute myocardial infarction (AMI) patients without DM [9-11]. However, little is known about the relationship between admission hyperglycemia and culprit plaque characteristics. Besides, some of these patients have undiagnosed DM $[12,13]$ which probably affects acute blood glucose (ABG) at admission. Thus, the acute-to-chronic glycemic ratio (A/C) [14], which is calculated by dividing $A B G$ by chronic blood glucose (CBG) estimated from $\mathrm{HbA}_{1 \mathrm{c}}$ [15], has been used to reflect relative hyperglycemia at admission.

In this study, we aim to investigate whether admission hyperglycemia in nondiabetic patients with AMI is associated with vulnerable culprit lesion characteristics such as plaque rupture, lipid-rich plaque, and TCFA. In addition, we compared different culprit plaque characteristics between diabetic and nondiabetic patients and evaluated two definitions for admission hyperglycemia for predicting vulnerable culprit plaque characteristics in AMI patients.

\section{Materials and Methods}

2.1. Study Population. The Optical Coherence Tomography Examination in Acute Myocardial Infarction (OCTAMI, NCT03593928) is a prospective, single-center, observational registry. In brief, consecutive patients at Fuwai Hospital were screened for OCT examination. The major inclusion criteria were (1) age $\geq 18$ years, (2) presented with ST-segment elevated myocardial infarction (STEMI), and (3) referred to primary percutaneous coronary intervention. The major exclusion criteria were (1) cardiogenic shock; (2) history of coronary artery bypass graft; (3) left main diseases, extremely tortuous or heavily calcified vessels; and (4) inability to obtain Thrombolysis in Myocardial Infarction Flow grade $\geq 2$. STEMI was defined as clinical symptoms, elevated troponin I level, and typical ST-segment elevation on electrocardiogram. In addition to the exclusion criteria of the OCTAMI registry, patients were excluded from the current study if (1) the culprit lesions were in-stent restenosis, coronary spasm, coronary embolism, or calcified nodule and (2) $\mathrm{HbA}_{1 \mathrm{c}}$ results were missing. All treatments were as per standard of care. Culprit vessel was determined primarily by coronary angiography and corroborated with electrocardiogram and echocardiographic results or ventriculographic assessments. All enrolled patients provided written informed consent. The study complied with the principles of the Declaration of Helsinki and was approved (no. 2017-866) by the Review Board of Fuwai Hospital.

2.2. Blood Glucose Measurements and Diagnosis of Diabetes. $\mathrm{ABG}$ was measured upon admission. $\mathrm{HbA}_{1 \mathrm{c}}$ was measured during hospital stay. DM was diagnosed if a patient had a history of diabetes, received insulin or oral hypoglycemic agents, or had $\mathrm{HbA}_{1 \mathrm{c}} \geq 6.5 \%(48 \mathrm{mmol} / \mathrm{mol})$. CBG was estimated from $\mathrm{HbA}_{1 \mathrm{c}}$ according to the following formula [15]: Estimated CBG $[\mathrm{mmol} / \mathrm{l}]=\{28.7 \times \mathrm{HbA} 1 \mathrm{c}[\%$ or $(\mathrm{mmol}$ $/ \mathrm{mol})]-46.7[\mathrm{mg} / \mathrm{dl}]\} / 18$. A/C was calculated as the ratio of $\mathrm{ABG}$ to estimated CBG. Two definitions for admission hyperglycemia were compared: (1) $A B G \geq 140 \mathrm{mg} / \mathrm{dl}$
(7.8 mmol/l) [16] and (2) A/C higher than the upper tertile cut-off of the current study population.

2.3. OCT Image Acquisition and Analysis. OCT examinations were performed as previously described [17, 18]. Briefly, OCT images of the culprit lesions were acquired using the frequency-domain OCT system (ILUMIEN OPTIS ${ }^{\mathrm{TM}}$, St. Jude Medical/Abbott, St. Paul, MN, USA) and a dragonfly catheter (Lightlab Imaging, Inc., Westford, MA, USA). Thrombus aspiration and/or gentle predilation were applied as per need. The total length of OCT pullback was $75 \mathrm{~mm}$. All OCT images were submitted to an offline St. Jude OCT Offline Review Workstation and analyzed by three independent investigators blinded to angiographic and clinical data. Any discordance was resolved by consensus. The culprit plaque was defined as the segment centered on the culprit lesion and extending bilaterally to $\geq 5 \mathrm{~mm}$ of normal vessel segment [19].

Definitions of image characteristics on OCT were based mainly on established consensus [20], and details of these definitions have been described previously [18]. Lipid was defined as a low-signal region with a poorly defined or diffuse border. Lipid arc was measured at $1 \mathrm{~mm}$ intervals across the entire lesion, and the largest arc was recorded. A plaque with a maximal lipid arc $>90^{\circ}$ was defined as a lipid-rich plaque (Figure S1b); otherwise, it was defined as a fibrous plaque (Figure S1a). Fibrous cap thickness (FCT) was measured in triplicate at the thinnest part of the fibrous cap of the culprit plaque, and the average value was calculated and reported. TCFA was defined as a lipid-rich plaque with FCT $<65 \mu \mathrm{m}$. Plaque rupture was defined as disruption of the fibrous cap with clear cavity formation (Figure S1c). Plaque erosion was defined based on evidence of thrombus on an irregular luminal surface without evidence of cap rupture in multiple adjacent frames (Figure S1d). Calcification was defined as signal-poor or heterogeneous regions with well-delineated borders (Figure S1e). Microvessels were defined as signal-poor, tubular structures without a connection to the vessel lumen in more than three consecutive cross-sectional images (Figure S1f). Cholesterol crystals were defined as linear structures with high backscatter within the plaque (Figure S1g). Macrophage infiltration was defined as signal rich, distinct, or confluent punctate regions above the intensity of background speckle noise with backward shadowing, usually located at the boundary between the fibrous cap and inner lipid core (Figure S1h). Thrombus was defined as an irregular mass floating in the lumen or adjacent to the luminal surface. The minimal lumen area (MLA) was the smallest lumen area within the length of the target lesion.

2.4. Statistical Analyses. Continuous variables with normal distribution are presented as mean $\pm \mathrm{SD}$ and compared between groups using the independent $t$-test; nonnormal variables were reported as median (interquartile range) and compared between groups using the Kruskal-Wallis test. Categorical variables are presented as number (\%) and compared between two groups by the $\chi^{2}$ test or the Fisher exact test. The $p_{\text {for trend }}$ were determined with a Wilcoxon type test 
for continuous variables and linear-by-linear association for categorical variables across ordered three $\mathrm{A} / \mathrm{C}$ tertile groups in non-DM patients. Bivariate correlations between OCT measurements including FCT, MLA, and maximal lipid arc (as continuous variables) and metabolic variables (as continuous variables) were analyzed using Spearman's correlation. Univariable and multivariable logistic regression analyses were applied to determine associations of OCT categorical characteristics (plaque rupture, lipid-rich plaque, and TCFA) and admission hyperglycemia. For admission hyperglycemia, we tested two different definitions by either $A B G$ or $A / C$, respectively, in univariable and multivariable logistic regression analyses. Covariates adjusted in the multivariable logistic regression model were age, sex, body mass index, current smoking, hypertension, baseline cardiac troponin I (cTnI), baseline creatine kinase $\mathrm{MB}$ (CK-MB), total cholesterol (TC), triglyceride (TG), low-density lipoprotein cholesterol (LDL-C) 1, high-density lipoprotein cholesterol (HDL-C), high-sensitive $\mathrm{C}$ reactive protein (hsCRP), estimated glomerular filtration rate (eGFR), previous statin usage, and time from symptom onset. A two-tailed $p$ value $<0.05$ was considered statistically significant. All analyses were performed using SPSS Statistics 24.0 software (IBM Corp, Armonk, NY) except that the Wilcoxon type test was performed using the $\mathrm{R}$ package rawr (version 0.9.1, Robert Redd, https://github.com/raredd/rawr; R Foundation for Statistical Computing, Vienna, Austria).

\section{Results}

Patients' baseline characteristics are summarized in Table 1. In total, 434 STEMI patients undergoing OCT examination from March 2017 to March 2019 were enrolled. Of these, 157 were excluded because of lack of preintervention OCT $(n=8)$, poor imaging quality $(n=83)$, in-stent restenosis $(n=34)$, coronary spasm $(n=11)$, coronary embolism $(n=2)$, calcified nodule $(n=17)$, and missing $\mathrm{HbA}_{1 \mathrm{c}}$ data $(n=2)$ (Figure 1). Table S1 (see supplementary material) shows the comparisons of baseline characteristics between included and excluded patients. Finally, 277 patients were included in the analysis, with $182(65.7 \%)$ nondiabetic patients and 95 (34.3\%) diabetic patients. Non-DM patients had lower ABG $(132.6 \mathrm{mg} / \mathrm{dl}$ (interquartile range 119.5$146.3)$ versus $211.5 \mathrm{mg} / \mathrm{dl}(177.2-271.3), p<0.001)$ and lower $\mathrm{HbA}_{1 \mathrm{c}}$ (5.7\% (5.5-5.9) versus 7.9\% (7.0-9.3), $p<$ $0.001)$ than DM patients but similar A/C (1.15 (1.03-1.27) versus $1.21(1.03-1.38), p>0.05)$. DM patients were heavier $(p<0.05)$ and had more multivessel disease $(p<0.05)$ than non-DM patients. Non-DM patients were further grouped by tertiles of $\mathrm{A} / \mathrm{C}$, with a lower tertile cut-off of 1.08 and upper tertile cut-off of 1.22. In non-DM patients, those in the highest $\mathrm{A} / \mathrm{C}$ tertile group were admitted to the hospital in the shortest time since symptom onset $\left(p_{\text {for trend }}=0.005\right)$. Across three $\mathrm{A} / \mathrm{C}$ tertiles in non-DM patients, there was a decreasing trend of cTnI ( $\left.p_{\text {fortrend }}=0.009\right)$ and CK-MB $\left(p_{\text {for trend }}=0.038\right)$. However, after adjustment for time from symptom onset, there was no linear association between $\mathrm{A} / \mathrm{C}$ tertiles and $\mathrm{CTnI}$ or between $\mathrm{A} / \mathrm{C}$ tertiles and CK-MB (Table S2).
Table 2 shows culprit lesion characteristics on OCT examination. Non-DM patients in the third A/C tertile group had the highest prevalence of plaque rupture (66.7\% versus $37.1 \%$ versus $38.3 \%$ from the third to the first tertile, $\left.p_{\text {for trend }}=0.002\right)$, lipid-rich plaque $(71.7 \%$ versus $46.8 \%$ versus $40.0 \%$ from the third to the first tertile, $\left.p_{\text {fortrend }}=0.001\right)$, and TCFA $(30.0 \%$ versus $21.0 \%$ versus $8.3 \%$ from the third to the first tertile, $p_{\text {for trend }}=0.003$ ) (Figure 2). Consistent with this, patients in the third A/C tertiles had the smallest FCT (median $90.0 \mathrm{~mm}$ versus $110.0 \mathrm{~mm}$ versus $110.0 \mathrm{~mm}$ from the third to the first tertile, $\left.p_{\text {fortrend }}=0.010\right)$. Rates of macrophage infiltration, microvessels, cholesterol crystal, calcification, and thrombus were similar among the $\mathrm{A} / \mathrm{C}$ tertiles in nondiabetic patients. While comparing the third $\mathrm{A} / \mathrm{C}$ tertile group of non-DM patients with DM patients, the prevalence of plaque rupture $\left(66.7 \%\right.$ versus $\left.56.8 \%, p_{3 \text { rd versus DM }}=0.222\right)$, lipid-rich plaque $\left(71.7 \%\right.$ versus $\left.68.4 \%, p_{3 \text { rd versus DM }}=0.669\right)$, and TCFA $\left(30.0 \%\right.$ versus $\left.34.7 \%, p_{3 \text { rd versus DM }}=0.406\right)$ was similar. The prevalence of calcification in DM patients was significantly higher than that of the third $\mathrm{A} / \mathrm{C}$ tertile group of non-DM patients $(p=0.002)$ but only numerically higher than that of the other two $\mathrm{A} / \mathrm{C}$ tertile groups of non-DM patients. On the other hand, the lowest A/C tertile of nonDM patients, compared with DM patients, had fewer macrophage infiltration $(p=0.049)$ and smaller FCT $(p=0.013)$. Overall, the DM group, compared with the non-DM group, had a significantly higher prevalence of lipid-rich plaque ( $68.4 \%$ versus $52.7 \%, p=0.012$ ), TCFA ( $34.7 \%$ versus $19.8 \%$, $p=0.006)$, cholesterol crystal $(12.6 \%$ versus $5.5 \%, p=0.037)$, and calcification $(62.1 \%$ versus $46.2 \%, p=0.012)$. However, the prevalence of plaque rupture was similar between DM patients and non-DM patients.

Table 3 shows the correlations between quantitative OCT measurements and glycemic markers and other laboratory variables in non-DM patients and DM patients. In non-DM patients, $\mathrm{ABG}$ and $\mathrm{A} / \mathrm{C}$ were correlated with $\mathrm{FCT}$ ( $r=-0.149, p=0.045 ; r=-0.206, p=0.005$, respectively). On the other hand, low-density lipoprotein cholesterol (LDL-C) and the estimated glomerular filtration rate (eGFR) were correlated with MLA $(r=-0.164, p=0.027 ; r=-0.229$, $p=0.002$, respectively) and lipid arc $(r=0.228, p=0.037$; $r=0.226, p=0.039$, respectively). In DM patients, no glycemic index was correlated with FCT, but total cholesterol (TC) and LDL-C were correlated with FCT ( $r=-0.216, p=0.036 ; r=-0.252, p=0.014$, respectively). All those correlations were significant but weak.

Table 4 shows results from univariate and multivariate logistic regression analyses of admission hyperglycemia for predicting plaque rupture, lipid-rich plaque, and TCFA in STEMI patients with or without DM, respectively. In non-DM patients, after adjustment for covariates, A/C $>1.22$ remained predictive for plaque rupture (hazard ratio (HR) 3.11, 95\% confidence interval (CI) 1.48-6.55, $p=0.003$ ), lipid-rich plaque (HR 2.94, 95\% CI $1.36-6.35, p=0.006$ ), and TCFA (HR 2.40, 95\% CI $1.01-5.72, p=0.049)$ but $\mathrm{ABG}>140 \mathrm{mg} / \mathrm{dl}$ was not predictive for plaque characteristics (Figure 3). In DM 


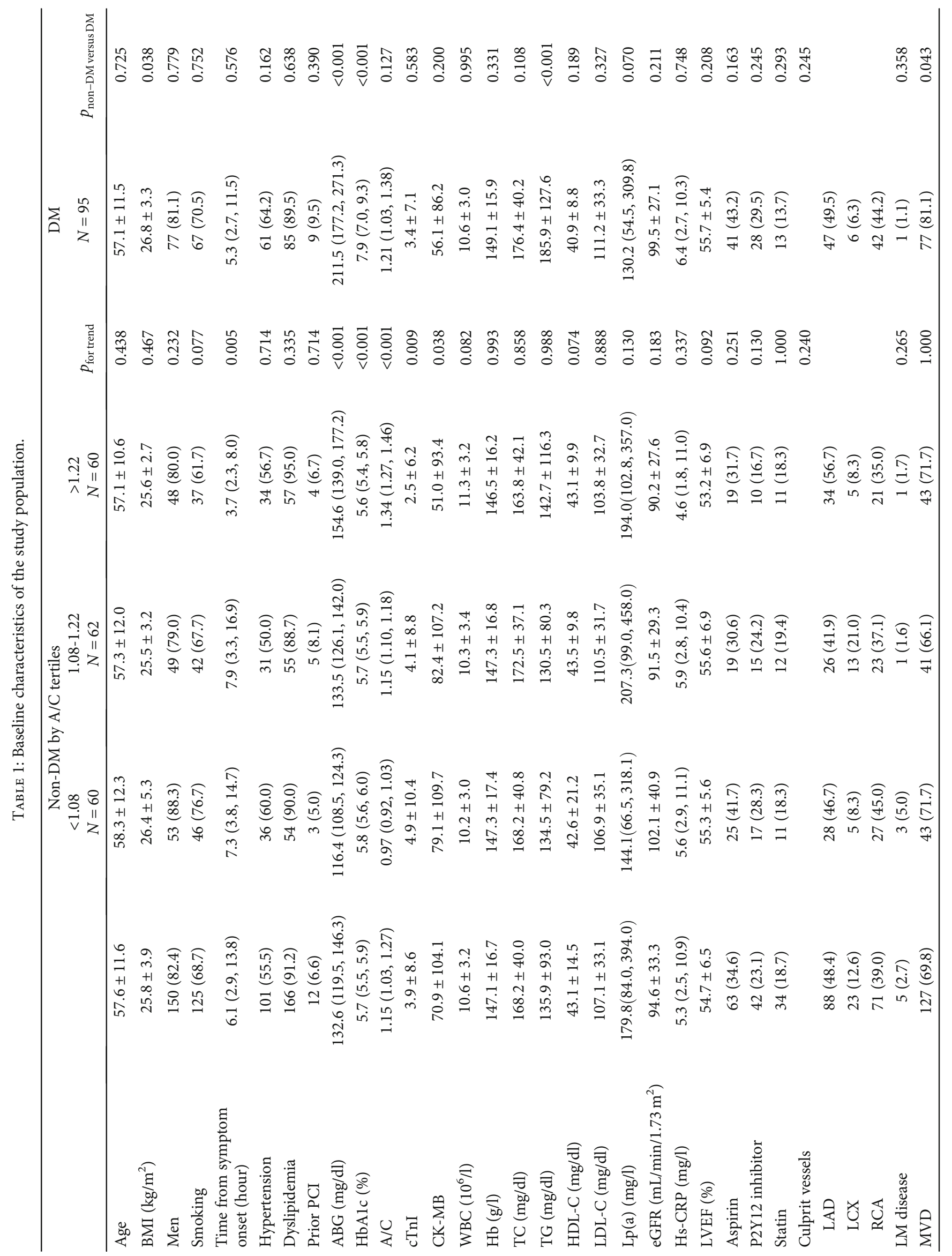




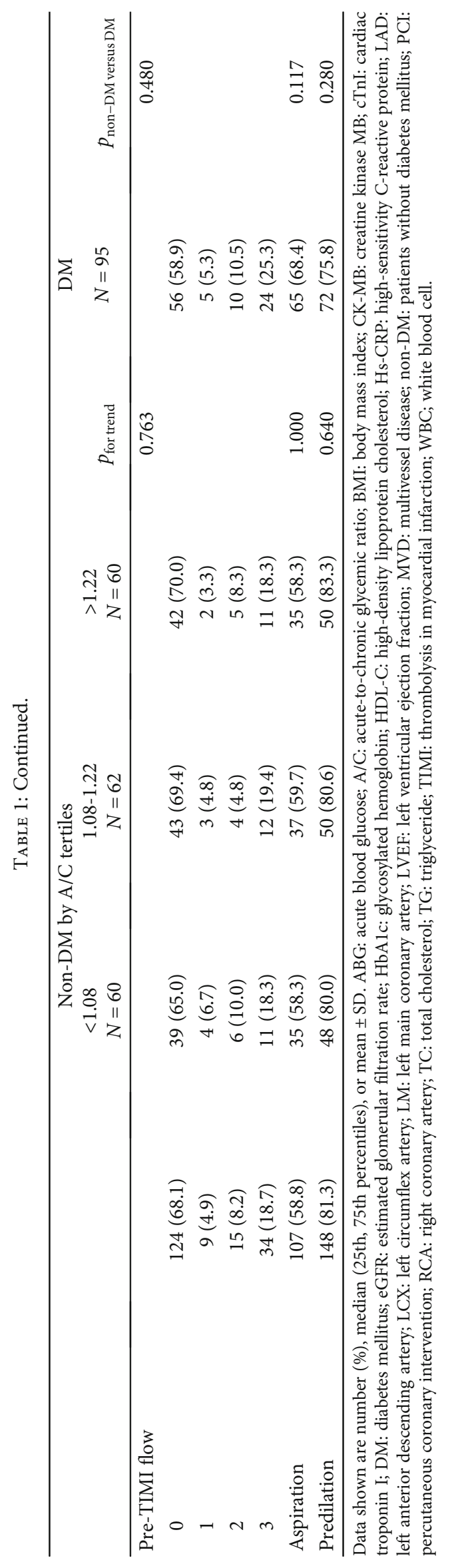




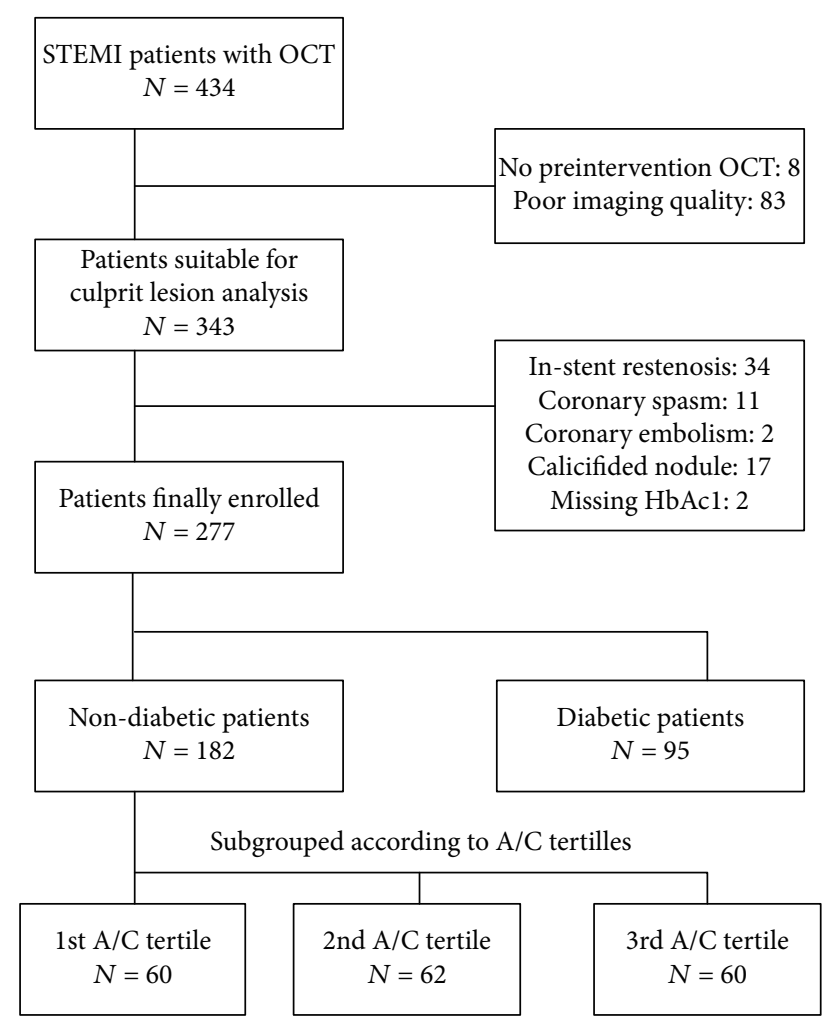

Figure 1: Flow chart. A/C: acute-to-chronic glycaemia ratio; HbAlc: hemoglobin Alc; OCT: optical coherence tomography; STEMI: ST-segment elevated myocardial infarction.

patients, both $\mathrm{A} / \mathrm{C}>1.22$ and $\mathrm{ABG}>140 \mathrm{mg} / \mathrm{dl}$ had no predictive value for plaque characteristics.

\section{Discussion}

Our study demonstrated that (1) DM patients with STEMI had a significantly higher prevalence of lipid-rich plaque, TCFA, cholesterol crystal, and calcification than non-DM patients; (2) non-DM STEMI patients with admission hyperglycemia had a higher prevalence of plaque rupture, lipidrich plaque, and TCFA at the culprit lesion than those without admission hyperglycemia; (3) admission hyperglycemia in non-DM patients defined as $\mathrm{A} / \mathrm{C}>1.22$ had a better predictive value for plaque rupture, lipid-rich plaque, and TCFA than that defined as ABG $>140 \mathrm{mg} / \mathrm{dl}$.

4.1. Culprit Lesion Characteristics in Diabetic STEMI. Different coronary and plaque characteristics between DM and non-DM patients have been reported. Previous studies showed larger lipid burden [21, 22], more plaque ruptures, and more TCFAs in diabetic patients than in nondiabetic patients [23]. Nicholls et al. [2] reported a strong relationship between percent atheroma volume and $\mathrm{HbA}_{1 \mathrm{c}}$. Several OCT studies $[3-6,8]$ had a limited sample size. The largest study [7] enrolled 322 patients with acute coronary syndrome and reported more lipid-rich plaque in the culprit lesion in the DM group than in the non-DM group. In addition, no difference in plaque rupture was observed between the groups [7]. Consistent with their study, our study showed more lipid-rich plaque and TCFA in DM patients than non-DM STEMI patients but a similar prevalence of plaque rupture in culprit lesion.

4.2. Culprit Lesion Characteristics in Nondiabetic STEMI. To our knowledge, this is the first study to compare plaque characteristics in non-DM patients according to $\mathrm{A} / \mathrm{C}$ tertiles. The current study showed that non-DM patients with the highest $\mathrm{A} / \mathrm{C}$ tertile had a higher prevalence of plaque rupture, lipidrich plaque, and TCFA than those in the other A/C tertiles. In consistence, non-DM patients with the highest $\mathrm{A} / \mathrm{C}$ tertile also had the smallest FCT. Surprisingly, the prevalence of plaque rupture, lipid-rich plaque, and TCFA was similar between non-DM patients in the highest $\mathrm{A} / \mathrm{C}$ tertile group and the $\mathrm{DM}$ group. Another recent study [18] from our group reported that AMI patients with increased duration of DM had a higher prevalence of plaque rupture, lipid-rich plaque, and TCFA along with higher HbAlc levels than those with short DM duration. In combination, these results suggested that hyperglycemia has a strong association with vulnerable plaque characteristics, namely, plaque rupture, lipid-rich plaque, and TCFA, regardless of DM status. It is worth mentioning that pretreatment with statin before acute coronary syndrome reduces not only the presentation of STEMI but also the prevalence of ruptured plaque and TCFA [24]. In the present study population, $18.7 \%$ of non-DM patients and 13.7\% DM patients were on statin therapy before the index STEMI $(p=0.293)$. Moreover, there was no difference of statin therapy across $\mathrm{A} / \mathrm{C}$ tertiles in non-DM patients. Thus, we do not consider statin pretreatment as a confounder between $\mathrm{A} / \mathrm{C}$ tertiles and plaque rupture or TCFA in non-DM patients.

Calcification is a long-term pathological change, and hyperglycemia promotes vascular calcification via multiple mechanisms such as oxidative stress, endothelial dysfunction, and advanced accumulation of glycation end products [25]. Moreover, race, sex, and age affect the prevalence of vascular calcification [26]. A previous OCT study showed an impact of chronic kidney diseases on coronary calcification [27]. The present study showed similar prevalence of calcification across three A/C tertiles but significantly fewer calcification in non-DM patients with the highest $\mathrm{A} / \mathrm{C}$ than in $\mathrm{DM}$ patients, which may result from the adjustment of those aforesaid confounders.

Coronary macrophage infiltration is another risk characteristic reflecting inflammatory level [22]. MacNeill et al. [28] reported more macrophage infiltration in unstable patients and culprit lesion than in stable patients and nonculprit lesions, respectively. The present study showed no difference in macrophage infiltration among $\mathrm{A} / \mathrm{C}$ tertiles in non-DM patients or between the DM and non-DM groups. However, those non-DM patients with the lowest A/C tertile had fewer macrophage infiltration than DM patients.

Although we could not make a cause-effective conclusion between admission hyperglycemia and culprit lesion characteristics, our hypothesis is that admission hyperglycemia represents severe myocardial infarction attack. Plaque rapture causes the sudden onset of acute myocardial infarction [29], and hormonal response to stress has a significant impact on hyperglycemia [30]. Then, hyperglycemia accelerate the 


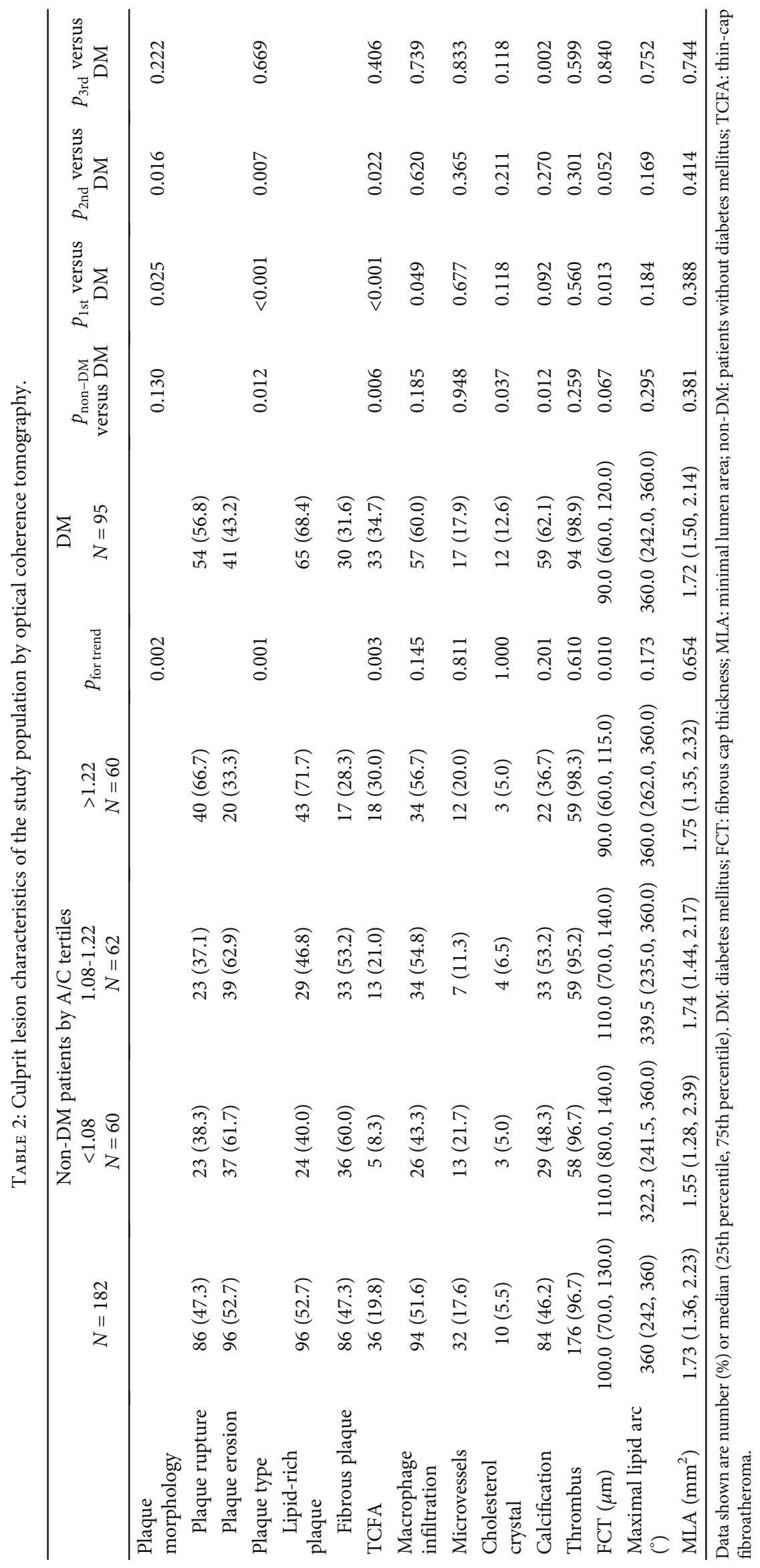




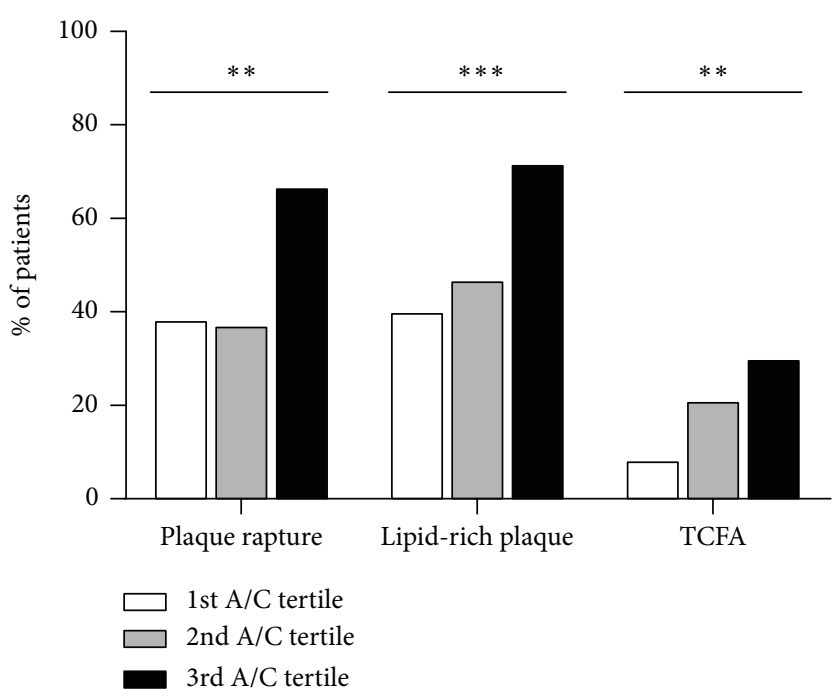

FIGURE 2: Comparison of vulnerable culprit lesion characteristics among A/C tertiles in nondiabetic patients with AMI, $n=182$. White bars: first A/C tertile, $n=60$; grey bars: second A/C tertile, $n=62$; black bars: third A/C tertile, $n=60 .{ }^{* *} p_{\text {for trend }} \leq 0.01,{ }^{* * *} p_{\text {for trend }} \leq 0.001$.

TABLE 3: Association between optical coherence tomography measurements and metabolic variables of the study population.

\begin{tabular}{lccccccccccc}
\hline & ABG & HbAlc & A/C & TC & TG & HDL-C & LDL-C & Lp (a) & Hs-CRP & eGFR \\
\hline Non-DM group & & & & & & & & & & \\
$\quad$ FCT, $n=182$ & $-0.149^{*}$ & 0.091 & $-0.206^{\dagger}$ & -0.138 & -0.045 & -0.019 & -0.122 & 0.097 & 0.143 & 0.065 \\
$\quad$ Lipid arc, $n=84^{\ddagger}$ & -0.038 & 0.006 & -0.019 & 0.193 & -0.026 & 0.092 & $0.228^{*}$ & 0.136 & -0.137 & $0.226^{*}$ \\
$\quad$ MLA, $n=182$ & 0.050 & 0.007 & 0.034 & -0.082 & 0.028 & 0.112 & $-0.164^{*}$ & 0.035 & 0.025 & $-0.229^{\dagger}$ \\
DM group & & & & & & & & & \\
$\quad$ FCT, $n=95$ & 0.157 & 0.158 & -0.029 & $-0.216^{*}$ & 0.012 & 0.031 & $-0.252^{*}$ & 0.194 & 0.154 & 0.108 \\
Lipid arc, $n=38^{\ddagger}$ & -0.022 & 0.011 & 0.071 & 0.073 & -0.116 & 0.059 & 0.079 & -0.096 & 0.014 & -0.105 \\
MLA, $n=95$ & 0.040 & 0.072 & 0.005 & -0.018 & 0.045 & -0.039 & -0.049 & -0.130 & 0.062 & 0.072 \\
\hline
\end{tabular}

${ }^{*} p<0.05 ;{ }^{\dagger} p<0.01 .{ }^{*}$ Individuals with lipid arc $=360^{\circ}$ were excluded, as truncated data is refused by Spearman's correlations. DM: diabetes mellitus.

vicious cycle of myocardial infarction by attenuated endothelium vasodilation [31], activated platelets [32], enhanced leukocyte accumulation [33], elevated inflammatory level [34], and increased thrombin generation potential [35]. In addition, previous studies reported that glycemic variability can promote atherosclerosis [36-39]. We also inferred that recurrent transient hyperglycemia before an index event has an accumulating impact on coronary atherosclerosis as well, but future studies are needed for evidence.

4.3. $A / C$ as a Predictor for Plaque Vulnerability in Nondiabetic AMI. Numerous glycemic metrics are available in clinical practice, but only a few are practical in a realworld acute setting of myocardial infarction. An early study by Capes et al. [40] reported an increased risk of death after AMI in all patients with high-glucose concentration on admission, which was described as stress hyperglycemia. However, it is plausible that AMI patients with the same ABG might have a different chronic glycemic metabolic status. Although chronic glucose level cannot be directly detected in AMI patients, it can be estimated from $\mathrm{HbA}_{1 \mathrm{c}}$ using the formula proposed by Nathan et al. [15]. Relative hyperglycemia or the stress hyperglycemia ratio, defined as ABG divided by estimated CBG (acute-to-chronic glucose ratio, A/C), was first proposed by Roberts et al. [14] as an improved biomarker of critical illness. Recent studies reported a prominent predictive value of $\mathrm{A} / \mathrm{C}$ for prognosis in AMI patients $[41,42]$. On the other hand, high-risk plaque is another independent prognostic predictor in patients with CAD (coronary artery disease) [43-46]. Thus, in AMI patients without DM, there may be an interrelation among acute hyperglycemia, vulnerable plaque, and poor prognosis. Our study showed a weak but significant correlation between ABG or A/C with FCT in nondiabetic patients.

Recent studies are exploring patient-tailored treatment strategy based on culprit lesion characteristics. EROSION Study (Effective Anti-Thrombotic Therapy Without Stenting: Intravascular Optical Coherence Tomography-Based Management in Plaque Erosion) [47] was the first study to suggest that patient with acute coronary syndrome due to plaque erosion can be managed by effective antiplatelet therapy without stent implantation. Torii et al. reported the impact of plaque type on stent strut [48]. However, high price and technical safety concerns restrict the application of OCT 
TABLE 4: Logistic regression analysis for plaque rupture, lipid plaque, and thin-cap fibroatheroma.

\begin{tabular}{|c|c|c|c|c|c|}
\hline & & $\begin{array}{c}\text { Univariable } \\
\text { OR }(95 \% \text { CI })\end{array}$ & $p$ values & $\begin{array}{l}\text { Multivariable } \\
\text { OR }(95 \% \mathrm{CI})\end{array}$ & $p$ values \\
\hline Non-DM & Plaque rupture & & & & \\
\hline \multirow[t]{8}{*}{$N=182$} & $\mathrm{ABG}>140 \mathrm{~m} / \mathrm{dl}$ & $1.67(0.90-3.11)$ & 0.105 & $1.65(0.80-3.38)$ & 0.174 \\
\hline & $\mathrm{A} / \mathrm{C}>1.22$ & $3.11(1.63-5.91)$ & $0.001^{\dagger}$ & $3.11(1.48-6.55)$ & $0.003^{\dagger}$ \\
\hline & Lipid-rich plaque & & & & \\
\hline & $\mathrm{ABG}>140 \mathrm{~m} / \mathrm{dl}$ & $1.62(0.87-3.03)$ & 0.130 & $1.69(0.80-3.56)$ & 0.169 \\
\hline & $\mathrm{A} / \mathrm{C}>1.22$ & $3.07(1.59-5.91)$ & $0.001^{\dagger}$ & $2.94(1.36-6.35)$ & $0.006^{\dagger}$ \\
\hline & Thin-cap fibroatheroma & & & & \\
\hline & $\mathrm{ABG}>140 \mathrm{~m} / \mathrm{dl}$ & $1.80(0.85-3.78)$ & 0.124 & $1.86(0.80-4.35)$ & 0.151 \\
\hline & $\mathrm{A} / \mathrm{C}>1.22$ & $2.40(1.14-5.04)$ & $0.021^{*}$ & $2.40(1.01-5.72)$ & $0.049^{*}$ \\
\hline DM & Plaque rupture & & & & \\
\hline \multirow[t]{8}{*}{$\mathrm{N}=95$} & $\mathrm{ABG}>140 \mathrm{~m} / \mathrm{dl}$ & $2.14(0.56-8.16)$ & 0.264 & $3.50(0.59-20.70)$ & 0.167 \\
\hline & $\mathrm{A} / \mathrm{C}>1.22$ & $1.28(0.57-2.89)$ & 0.556 & $1.00(0.37-2.72)$ & 0.994 \\
\hline & Lipid-rich plaque & & & & \\
\hline & $\mathrm{ABG}>140 \mathrm{~m} / \mathrm{dl}$ & $0.92(0.22-3.83)$ & 0.910 & $0.71(0.10-5.13)$ & 0.732 \\
\hline & $\mathrm{A} / \mathrm{C}>1.22$ & $1.04(0.44-2.48)$ & 0.926 & $0.95(0.31-2.86)$ & 0.923 \\
\hline & Thin-cap fibroatheroma & & & & \\
\hline & $\mathrm{ABG}>140 \mathrm{~m} / \mathrm{dl}$ & $1.27(0.31-5.29)$ & 0.740 & $0.92(0.12-6.82)$ & 0.934 \\
\hline & $\mathrm{A} / \mathrm{C}>1.22$ & $1.29(0.55-3.00)$ & 0.555 & $1.12(0.36-3.44)$ & 0.847 \\
\hline
\end{tabular}

${ }^{*} p<0.05 ;{ }^{\dagger} p<0.01$. ABG: acute blood glucose; A/C: acute versus chronic; CI: confidence interval; DM: diabetes mellitus; OR: odds ratio.

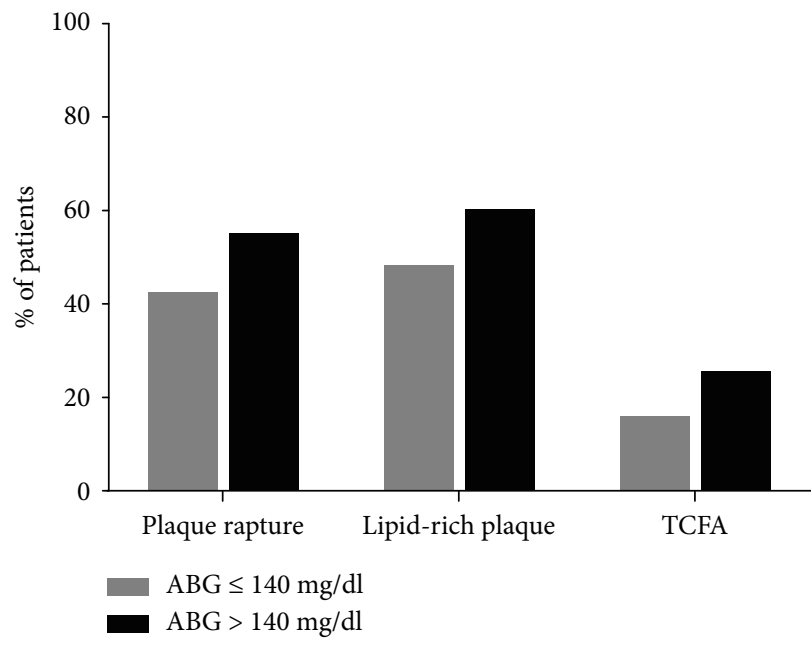

(a)

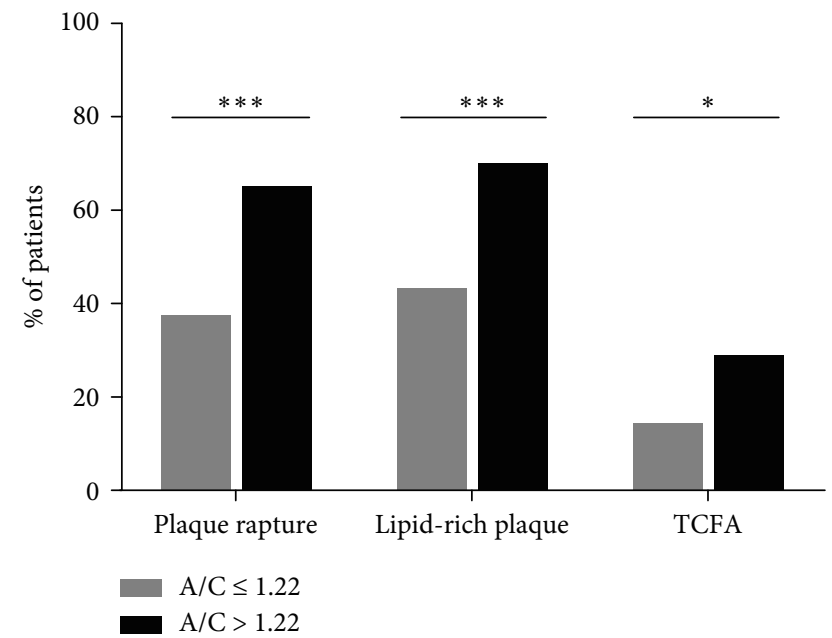

(b)

FIgURE 3: Comparisons of vulnerable culprit lesion characteristics by different hyperglycemia criteria in non-diabetic patients with AMI, $n=182$. (a) Hyperglycemia defined as A/C > 1.22; (b) hyperglycemia defined as ABG > $140 \mathrm{mg} / \mathrm{dl}$. Gray bars: no hyperglycemia; black bars: hyperglycemia. Significant difference: ${ }^{*} p \leq 0.05,{ }^{* * *} p \leq 0.001$.

to a selected population of AMI, surrogates for predicting culprit lesions are needed in clinical practice. Our results showed that $\mathrm{A} / \mathrm{C}$ ratio had a significant association with vulnerable plaque characteristics. Moreover, admission hyperglycemia defined by $\mathrm{A} / \mathrm{C}>1.22$ was associated with a high risk of plaque rupture, lipid-rich plaque, and TCFA, but admission hyperglycemia defined by ABG $>140 \mathrm{mg} / \mathrm{dl}$ had no predictive value. Thus, $\mathrm{A} / \mathrm{C}$ had a better predictive ability than ABG alone for plaque vulnerability, which might be an important message to physicians in clinical practice.

4.4. Study Limitations. Some limitations warrant mention. First, this is an observational study with prospectively enrolled patients and retrospectively collected data, and therefore, no cause-effect relationship between hyperglycemia and vulnerable plaque characteristics could be established. Second, OCT 
examination is restricted to patients with relatively stable hemodynamics for ethical and safety reasons, and thus, selection bias could not be eliminated which undoubtedly contributed to the percent $(50.5 \%)$ of plaque rupture in our study population being much lower than that (70\%) reported in a meta-analysis by Iannaccone et al. [49]. Third, undiagnosed DM is not rare in AMI. Thus, we carefully screened for DM history and $\mathrm{HbA}_{1 \mathrm{c}}$. In addition, we applied $\mathrm{A} / \mathrm{C}$ in the non-DM group, which had taken into consideration both $\mathrm{ABG}$ and $\mathrm{CBG}$ and were available in an acute setting of AMI. Fourth, due to the limited number of patients in our study cohort, we cannot extend our conclusions to all AMI patients. Therefore, further studies are needed.

\section{Conclusions}

This study demonstrated that non-DM patients with admission hyperglycemia had a higher prevalence of vulnerable culprit plaque characteristics than those without admission hyperglycemia. Moreover, A/C was more valuable than $\mathrm{ABG}$ in predicting culprit plaque vulnerability in non-DM patients with AMI. These findings highlight the important role of admission hyperglycemia in non-DM patients with AMI. Future studies are needed to improve the glucose evaluation and management strategies which may consequently improve clinical outcomes in these patients.

\section{Data Availability}

The data that support the findings of this study are available from the corresponding author upon reasonable request.

\section{Disclosure}

Dr Yan had full access to all the data in the study and takes responsibility for its integrity and the data analysis.

\section{Conflicts of Interest}

All authors declare no conflict of interest regarding the publication of this paper.

\section{Acknowledgments}

We thank all participants for their support of this study. This work was supported by Chinese Academy of Medical Sciences Innovation Fund for Medical Sciences (grant number 2016-I2M-1-009).

\section{Supplementary Materials}

Table S1: baseline characteristics of the study population and excluded patients. Table S2: linear regression analysis between $\mathrm{A} / \mathrm{C}$ and $\mathrm{CTnI} / \mathrm{CK}-\mathrm{MB}$. Figure $\mathrm{S} 1$ : representative cross-sectional optical coherence tomography images. (Supplementary Materials)

\section{References}

[1] Emerging Risk Factors Collaboration, "Diabetes mellitus, fasting blood glucose concentration, and risk of vascular disease: a collaborative meta-analysis of 102 prospective studies," Lancet, vol. 375, no. 9733, pp. 2215-2222, 2010.

[2] S. J. Nicholls, E. M. Tuzcu, S. Kalidindi et al., "Effect of diabetes on progression of coronary atherosclerosis and arterial remodeling: a pooled analysis of 5 intravascular ultrasound trials," Journal of the American College of Cardiology, vol. 52, no. 4, pp. 255-262, 2008.

[3] S. Chia, O. C. Raffel, C. M. Takano, G. J. Tearney, B. E. Bouma, and I. K. Jang, "Comparison of coronary plaque characteristics between diabetic and non- diabetic subjects: An in vivo optical coherence tomography study," Diabetes Research and Clinical Practice, vol. 81, no. 2, pp. 155-160, 2008.

[4] T. Feng, C. Yundai, C. Lian et al., "Assessment of coronary plaque characteristics by optical coherence tomography in patients with diabetes mellitus complicated with unstable angina pectoris," Atherosclerosis, vol. 213, no. 2, pp. 482-485, 2010.

[5] G. Niccoli, S. Giubilato, L. di Vito et al., "Severity of coronary atherosclerosis in patients with a first acute coronary event: a diabetes paradox," European Heart Journal, vol. 34, no. 10, pp. 729-741, 2013.

[6] K. Kato, T. Yonetsu, S. J. Kim et al., "Comparison of nonculprit coronary plaque characteristics between patients with and without diabetes: a 3-vessel optical coherence tomography study," JACC: Cardiovascular Interventions, vol. 5, no. 11, pp. 1150-1158, 2012.

[7] T. Sugiyama, E. Yamamoto, K. Bryniarski et al., "Coronary plaque characteristics in patients with diabetes mellitus who presented with acute coronary syndromes," Journal of the American Heart Association, vol. 7, no. 14, article e009245, 2018.

[8] T. Yonetsu, K. Kato, S. Uemura et al., "Features of coronary plaque in patients with metabolic syndrome and diabetes mellitus assessed by 3-vessel optical coherence tomography," Circulation: Cardiovascular Imaging, vol. 6, no. 5, pp. 665-673, 2013.

[9] A. Norhammar, Å. Tenerz, G. Nilsson et al., "Glucose metabolism in patients with acute myocardial infarction and no previous diagnosis of diabetes mellitus: a prospective study," Lancet, vol. 359, no. 9324, pp. 2140-2144, 2002.

[10] M. Ishihara, I. Inoue, T. Kawagoe et al., "Is admission hyperglycaemia in non-diabetic patients with acute myocardial infarction a surrogate for previously undiagnosed abnormal glucose tolerance?," European Heart Journal, vol. 27, no. 20, pp. 2413-2419, 2006.

[11] C. K. Naber, R. H. Mehta, C. Jünger et al., "Impact of admission blood glucose on outcomes of nondiabetic patients with acute ST-elevation myocardial infarction (from the German Acute Coronary Syndromes [ACOS] Registry)," The American Journal of Cardiology, vol. 103, no. 5, pp. 583-587, 2009.

[12] R. Doerr, U. Hoffmann, W. Otter et al., "Oral glucose tolerance test and $\mathrm{HbAlc}$ for diagnosis of diabetes in patients undergoing coronary angiography the Silent Diabetes Study," Diabetologia, vol. 54, no. 11, pp. 2923-2930, 2011.

[13] V. Gyberg, D. de Bacquer, K. Kotseva et al., "Screening for dysglycaemia in patients with coronary artery disease as reflected by fasting glucose, oral glucose tolerance test, and HbAlc: a report from EUROASPIRE IV-a survey from the European Society of Cardiology," European Heart Journal, vol. 36, no. 19, pp. 1171-1177, 2015.

[14] G. W. Roberts, S. J. Quinn, N. Valentine et al., "Relative hyperglycemia, a marker of critical illness: introducing the stress 
hyperglycemia ratio," The Journal of Clinical Endocrinology and Metabolism, vol. 100, no. 12, pp. 4490-4497, 2015.

[15] D. M. Nathan, J. Kuenen, R. Borg, H. Zheng, D. Schoenfeld, and R. J. Heine, "Translating the A1C assay into estimated average glucose values," Diabetes Care, vol. 31, no. 8, pp. 1473-1478, 2008.

[16] P. Deedwania, M. Kosiborod, E. Barrett et al., "Hyperglycemia and acute coronary syndrome: a scientific statement from the American Heart Association Diabetes Committee of the Council on nutrition, physical activity, and metabolism," Circulation, vol. 117, no. 12, pp. 1610-1619, 2008.

[17] Y. Tan, Z. Sheng, P. Zhou et al., "Plasma trimethylamine Noxide as a novel biomarker for plaque rupture in patients with ST-segment-elevation myocardial infarction," Circulation: Cardiovascular Interventions, vol. 12, no. 1, article e007281, 2019.

[18] Z. Sheng, P. Zhou, C. Liu et al., "Relationships of coronary culprit-plaque characteristics with duration of diabetes mellitus in acute myocardial infarction: an intravascular optical coherence tomography study," Cardiovascular Diabetology, vol. 18, no. 1, p. 136, 2019.

[19] J. Tian, X. Ren, R. Vergallo et al., "Distinct morphological features of ruptured culprit plaque for acute coronary events compared to those with silent rupture and thin-cap fibroatheroma: a combined optical coherence tomography and intravascular ultrasound study," Journal of the American College of Cardiology, vol. 63, no. 21, pp. 2209-2216, 2014.

[20] G. J. Tearney, E. Regar, T. Akasaka et al., "Consensus standards for acquisition, measurement, and reporting of intravascular optical coherence tomography studies: a report from the International Working Group for Intravascular Optical Coherence Tomography Standardization and Validation," Journal of the American College of Cardiology, vol. 59, no. 12, pp. 1058-1072, 2012.

[21] A. P. Burke, F. D. Kolodgie, A. Zieske et al., "Morphologic findings of coronary atherosclerotic plaques in Diabetics," Arteriosclerosis, Thrombosis, and Vascular Biology, vol. 24, no. 7, pp. 1266-1271, 2004.

[22] P. R. Moreno, A. M. Murcia, I. F. Palacios et al., "Coronary composition and macrophage infiltration in atherectomy specimens from patients with diabetes mellitus," Circulation, vol. 102, no. 18, pp. 2180-2184, 2000.

[23] Y. J. Hong, M. H. Jeong, Y. H. Choi et al., "Plaque characteristics in culprit lesions and inflammatory status in diabetic acute coronary syndrome patients," JACC: Cardiovascular Imaging, vol. 2, no. 3, pp. 339-349, 2009.

[24] S. Gili, M. Iannaccone, F. Colombo et al., "Effects of statins on plaque rupture assessed by optical coherence tomography in patients presenting with acute coronary syndromes: insights from the optical coherence tomography (OCT)-FORMIDABLE registry," European Heart Journal Cardiovascular Imaging, vol. 19, no. 5, pp. 524-531, 2018.

[25] K. Yahagi, F. D. Kolodgie, C. Lutter et al., "Pathology of human coronary and carotid artery atherosclerosis and vascular calcification in diabetes mellitus," Arteriosclerosis, Thrombosis, and Vascular Biology, vol. 37, no. 2, pp. 191-204, 2017.

[26] R. L. McClelland, H. Chung, R. Detrano, W. Post, and R. A. Kronmal, "Distribution of coronary artery calcium by race, gender, and age: results from the Multi-Ethnic Study of Atherosclerosis (MESA)," Circulation, vol. 113, no. 1, pp. 30-37, 2006.
[27] T. Sugiyama, S. Kimura, H. Ohtani et al., "Impact of chronic kidney disease stages on atherosclerotic plaque components on optical coherence tomography in patients with coronary artery disease," Cardiovascular Intervention and Therapeutics, vol. 32, no. 3, pp. 216-224, 2017.

[28] B. D. MacNeill, I.-K. Jang, B. E. Bouma et al., "Focal and multifocal plaque macrophage distributions in patients with acute and stable presentations of coronary artery disease," Journal of the American College of Cardiology, vol. 44, no. 5, pp. 972979, 2004.

[29] S. Kojima, H. Nonogi, Y. Miyao et al., "Is preinfarction angina related to the presence or absence of coronary plaque rupture?" Heart, vol. 83, no. 1, pp. 64-68, 2000.

[30] G. A. Oswald, C. C. T. Smith, D. J. Betteridge, and J. S. Yudkin, "Determinants and importance of stress hyperglycaemia in non-diabetic patients with myocardial infarction," British Medical Journal (Clinical Research Ed), vol. 293, no. 6552, pp. 917-922, 1986.

[31] S. B. Williams, A. B. Goldfine, F. K. Timimi et al., "Acute hyperglycemia attenuates endothelium-dependent vasodilation in humans in vivo," Circulation, vol. 97, no. 17, pp. 1695-1701, 1998.

[32] P. Gresele, G. Guglielmini, M. de Angelis et al., "Acute, shortterm hyperglycemia enhances shear stress-induced platelet activation in patients with type II diabetes mellitus," Journal of the American College of Cardiology, vol. 41, no. 6, pp. 1013-1020, 2003.

[33] Y. H. Jason, S. R. Leslie, D. Grace, D. C. Adriana, G. C. Jack, and F. M. Paul, "Diabetes enhances leukocyte accumulation in the coronary microcirculation early in reperfusion following ischemia," Journal of Diabetes and its Complications, vol. 14, pp. 96-107, 2000.

[34] K. Esposito, F. Nappo, R. Marfella et al., "Inflammatory cytokine concentrations are acutely increased by hyperglycemia in humans: role of oxidative stress," Circulation, vol. 106, no. 16, pp. 2067-2072, 2002.

[35] S. Lee, C. Ay, C. W. Kopp, S. Panzer, and T. Gremmel, "Impaired glucose metabolism is associated with increased thrombin generation potential in patients undergoing angioplasty and stenting," Cardiovascular Diabetology, vol. 17, no. 1, p. 131, 2018.

[36] S. Kataoka, M. Gohbara, N. Iwahashi et al., "Glycemic variability on continuous glucose monitoring system predicts rapid progression of non-culprit lesions in patients with acute coronary syndrome," Circulation Journal, vol. 79, no. 10, pp. 22462254, 2015.

[37] M. Kuroda, T. Shinke, K. Sakaguchi et al., “Association between daily glucose fluctuation and coronary plaque properties in patients receiving adequate lipid-lowering therapy assessed by continuous glucose monitoring and optical coherence tomography," Cardiovascular Diabetology, vol. 14, no. 1, p. 78, 2015.

[38] K. Okada, K. Hibi, M. Gohbara et al., "Association between blood glucose variability and coronary plaque instability in patients with acute coronary syndromes," Cardiovascular Diabetology, vol. 14, no. 1, p. 111, 2015.

[39] M. Gohbara, K. Hibi, T. Mitsuhashi et al., "Glycemic variability on continuous glucose monitoring system correlates with non-culprit vessel coronary plaque vulnerability in patients with first-episode acute coronary syndrome - Optical Coherence Tomography Study," Circulation Journal, vol. 80, no. 1, pp. 202-210, 2016. 
[40] S. E. Capes, D. Hunt, K. Malmberg, and H. C. Gerstein, "Stress hyperglycaemia and increased risk of death after myocardial infarction in patients with and without diabetes: a systematic overview," Lancet, vol. 355, no. 9206, pp. 773-778, 2000.

[41] G. Marenzi, N. Cosentino, V. Milazzo et al., "Prognostic value of the acute-to-chronic glycemic ratio at admission in acute myocardial infarction: a prospective study," Diabetes Care, vol. 41, no. 4, pp. 847-853, 2018.

[42] Y. Yang, T. H. Kim, K. H. Yoon et al., "The stress hyperglycemia ratio, an index of relative hyperglycemia, as a predictor of clinical outcomes after percutaneous coronary intervention," International Journal of Cardiology, vol. 241, pp. 57-63, 2017.

[43] S.-E. Lee, J. M. Sung, D. Andreini et al., "Differences in progression to obstructive lesions per high-risk plaque features and plaque volumes with CCTA," JACC: Cardiovascular Imaging, vol. 13, no. 6, pp. 1409-1417, 2020.

[44] M. Ferencik, T. Mayrhofer, D. O. Bittner et al., "Use of highrisk coronary atherosclerotic plaque detection for risk stratification of patients with stable chest pain: a secondary analysis of the PROMISE randomized clinical trial," Journal of the American College of Cardiology, vol. 3, no. 2, pp. 144-152, 2018.

[45] S. Motoyama, H. Ito, M. Sarai et al., "Plaque characterization by coronary computed tomography angiography and the likelihood of acute coronary events in mid-term follow-up," Journal of the American College of Cardiology, vol. 66, no. 4, pp. 337-346, 2015.

[46] F. Prati, E. Romagnoli, L. Gatto et al., "Relationship between coronary plaque morphology of the left anterior descending artery and 12 months clinical outcome: the CLIMA study," European Heart Journal, vol. 41, pp. 383-391, 2019.

[47] L. Xing, E. Yamamoto, T. Sugiyama et al., "EROSION Study (Effective Anti-Thrombotic Therapy Without Stenting: Intravascular Optical Coherence Tomography-Based Management in Plaque Erosion): a 1-year follow-up report," Circulation: Cardiovascular Interventions, vol. 10, no. 12, 2017.

[48] R. Torii, E. Tenekecioglu, Y. Katagiri et al., "The impact of plaque type on strut embedment/protrusion and shear stress distribution in bioresorbable scaffold," European Heart Journal Cardiovascular Imaging, vol. 21, no. 4, pp. 454-462, 2020.

[49] M. Iannaccone, G. Quadri, S. Taha et al., "Prevalence and predictors of culprit plaque rupture at OCT in patients with coronary artery disease: a meta-analysis," European Heart Journal Cardiovascular Imaging, vol. 17, no. 10, pp. 11281137, 2016. 